\title{
Efficient Multi-Level Access Control for Geo-Contents Maintenance
}

\author{
Sun-Rae PARK ${ }^{1}$, Jong Suk RUTH LEE ${ }^{1}, \mathrm{Kum} \mathrm{Won} \mathrm{CHO}^{1}, \mathrm{Kyu}^{-C h u l ~ \mathrm{LE}^{2}}$ \\ ${ }^{1}$ Cyber Environment Development Department, Supercomputing Center, \\ Korea Institute of Science and Technology Information, \\ 245 Daehak-ro, Yuseong-gu, Daejeon, 305-806, Korea, \\ \{srpark, jsruthlee, ckw\}@kisti.re.kr \\ 2 Department of Computer Engineering at Chungnam National University, \\ 99 Daehak-ro, Yuseong-gu, Daejeon , 305-764, Korea, \\ kclee@cnu.ac.kr
}

\begin{abstract}
GIS data is digital content that requires high initial production cost and it is transformed into different digital content according to its usage or access level. The convenience of digital content has increased the demands of the content, but because of characteristic of digital content which can be duplicated unlimitedly, the issues on security and copyright have been raised frequently. This paper proposes multi-level access control using Delta Compress Algorithm and by granting access control based on usages and permissions of content, it prevents the illegal use of GIS data which will protect and maintain digital contents consistently. Multi-Level Access Control using Delta Compress proposed in this paper is applied to all digital content in the same way and the same application is possible to Raster Image without additional work for file format.
\end{abstract}

Keywords: Geo-Contents, DRM, Vector data, Raster data, Delta compress

\section{Introduction}

The development of the Internet and network has enabled real-time services that provide a huge amount of high quality digital content [1]. As digital technology and communication technology are becoming more common, end users can create, modify and distribute digital content with little effort. The convenience of digital content has increased the demands of the content, but because of characteristic of digital content which can be duplicated unlimitedly, the issues on security and copyright[2][12] have been raised frequently. Especially, GIS data has been extended to Geo-Contents concept which includes digital contents related to geographic information and it is expanded into many different forms of data.[9]-[11]

Copyright protection is needed because of the high initial production cost and importance of the information. Due to the nature of security in GIS data (classified into closed, open, and restricted), access to GIS data is different based on its usage and access level.

This paper proposes a Multi-level access control technique to protect the copyright of Geo-Content which provides in various forms and to control the access of Geo-Content based on access level. When Multi-Level Access Control has accesses and uses Geo-Content, it provides access to Geo-Content that corresponds to its access level. Multi-Level Access Control technique using Delta
Compress Algorithm is also easily applied to GIS data that is provided in various forms. In particularly, it can be applicable to Vector data and Raster data as well as file formats which do not have layer and object.

\section{Multi-level Access Control}

\subsection{GIS data feature}

GIS data are categorized into two main forms: vector data and raster data. Vector data consist of points, lines and polygons. Vector data can be represented without any loss of accuracy and quality at any scale. Text font area is one general type of vector data. Vector data contain spatial data to be used in GIS.

Although vector data have been widely used, no agreement on the global standard format has been made yet, and shape file format has been used as a nonofficial standard for vector data in GIS. Open GIS Consortium (OGC) adopted Keyhole Markup Language (KML) of Google as a standard for vector data.

Raster data are grid cells that contain elevations, zoning, or images. Since raster data have specific resolution, they can be degraded at different scale. A common type of raster data is JPEG. GIS images are usually tied with a common special data or geo-rectified.

Examples of raster data are aerial imagery and LiDAR. 
The Ubiquitous technology extends GIS data to geocontent that may contain various types of data including multimedia content related to geographic information.

\subsection{Delta compress algorithm}

Delta Compression is a compression technique without losing any information which is used to update software or source codes. This differential compression depends on the similarities between two files, and thus results in a higher compression rate than any other compression method. This technique is very efficient and useful for data synchronization between connected computers in a network with low bit rates[4].

In this paper, Delta Compression System, a compressor uses two files as input; one is a target file to compress, the other is a reference file or a basis file. The delta compressor can represent the target file more compactly. The compact representation or delta may include references to similar data found in the basis file. The Delta de-compressor, or applier, accepts the delta along with the basis file, and reconstructs the target file.

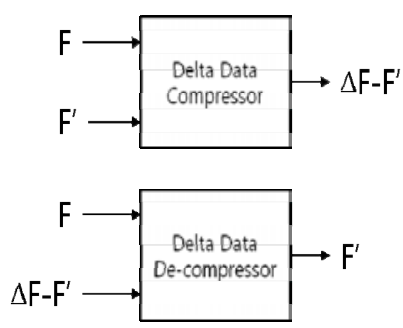

Figure 1. Delta Compression process

Figure 1 illustrates the Delta Compression process. The delta creator accepts target data F' along with basis $\mathrm{F}$, and produces a compressed representation $\Delta(F, F)$. Later, the delta applier accepts the delta $\Delta\left(F, F^{\prime}\right)$ along with the same basis $\mathrm{F}$, and reconstructs the target data F'.

$$
\begin{aligned}
& \operatorname{size}\left(\Delta\left(F, F^{\prime}\right)\right)<\operatorname{size}\left(F^{\prime}\right) \\
& \operatorname{size}\left(\Delta\left(F, F^{\prime}\right)\right)<\operatorname{size}\left(C(F)^{\prime}\right)
\end{aligned}
$$

Because the delta file seeks and compact the differences between two data, the size of the delta file will decrease more if the original file $F$ and the target file $F$ ' are similar. On the contrary, if no differences between the original file $\mathrm{F}$ and the target file $\mathrm{F}$ ' are found, the size of the delta can rather increase. In this case, it is more efficient to compress the target file F' alone [3]-[8].
Delta Compression Algorithm looks into the differences between two binary files but not text files, so the algorithm is applicable to all data types.

\subsection{Multi-level access control schema}

The proposed Multi-level access control is a technique that controls access to Geo-Content based on its usage and access level. It also enables control access to the resources not only in server areas but also in local personal computers.

Multi-Level Access Control for Geo-Contents in GIS must satisfy following conditions.

- Support various file formats.

- Support layer-level access control.

- Support object-level access control.

- Support access control for Vector Data as well as Raster Data.

Figure 2 illustrates the concept of the layerlevel access control. It grants access to specific layers in GIS data consisting of a number of layers based on its usage. As shown in figure, different scenarios for the GIS data consisting of layer 0 , layer 1 , layer 2 and layer 4 may exist as follows.

1. All the layers are used.

2. Two layers, layer 2 and 4 are used.

3. Only layer 1 is used.

In the three scenarios of layer access control, layers which had access to use were all the same layers but it granted three different access to the layers based on the usage.

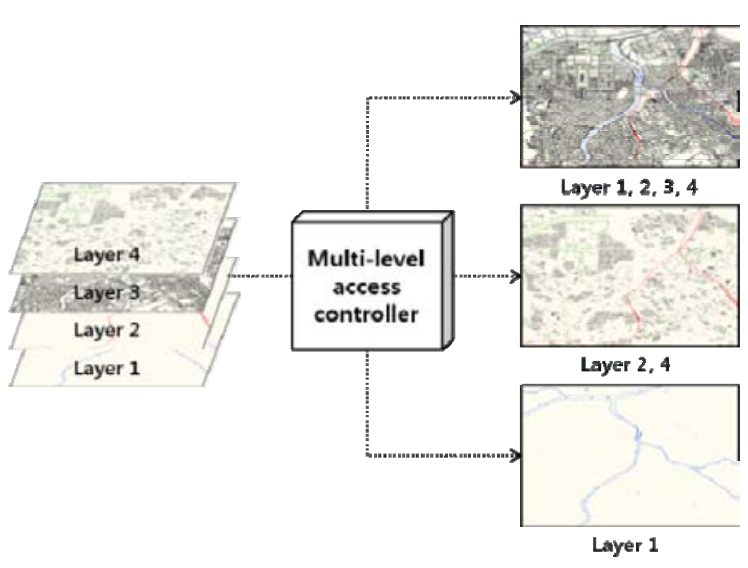

Figure 2. Layer access control

Figure 3 explains the concept of object-level access control and possible scenarios. The 
access to the objects in the GIS data is granted according to user classes. For the GIS data consisting of a rectangle of class 0 object and diamonds of class 1 object, the following scenarios can exist.

1. In case of access control for class 0 objects, a user of class 0 is authorized to access all the objects.

2. In case of access control for class 1 objects, a user of class 1 is authorized to access all the diamonds of class 1 object except the rectangle of class 0 object.

3. A user of class 2 is not authorized to access any objects in diamonds of class 1 and rectangle of class 0 objects.

In three scenarios of object access control, objects were granted or denied access depending on their access level.

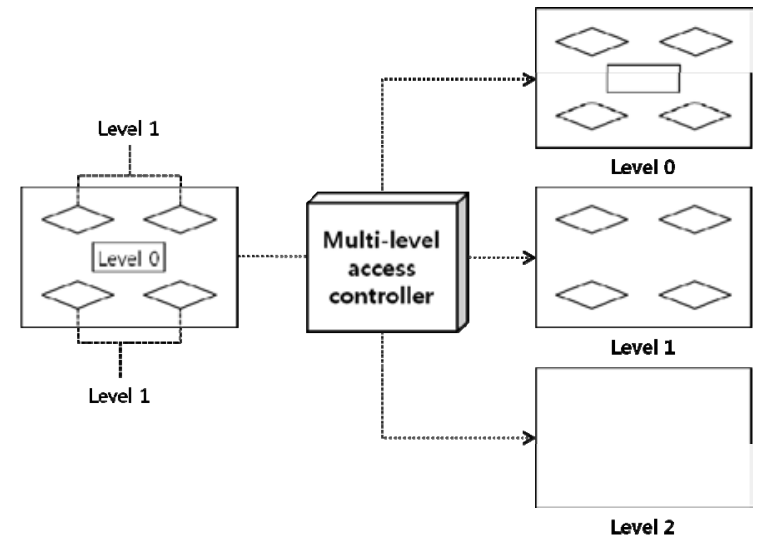

Figure 3. Object access control

\section{Multi-level Access Control Implementation}

To provide the access control to individual objects and layers in GIS data, the highest-level data and the Delta, which is compressed by extracting from the differences between the highest-class and lower-class GIS data, are packaged in a Geo-Content together. The users receiving such packaged Geo-Content have to be licensed to be able to gain usage and access level.

When Geo-Processing techniques are used to control access to Geo-Content, an engine that can interpret the file format used in the GeoContent is needed. For example, to control access to Shapefile, we need an engine interpreting the Shapefile format. Similarly, to control access to drawing file, we need an engine which can interpret the drawing file format. It means that engines that are able to interpret the files should be added to file formats which would increase the control access. In addition, image processing techniques are also required to control access to Raster Data, instead of Geo-Processing technique. However, the access control using Delta Compress Algorithm can overcome such problems. The access control using Delta compress algorithm can be directly applied to all file format such as Vector Data, Raster Data and its related multi-media contents.

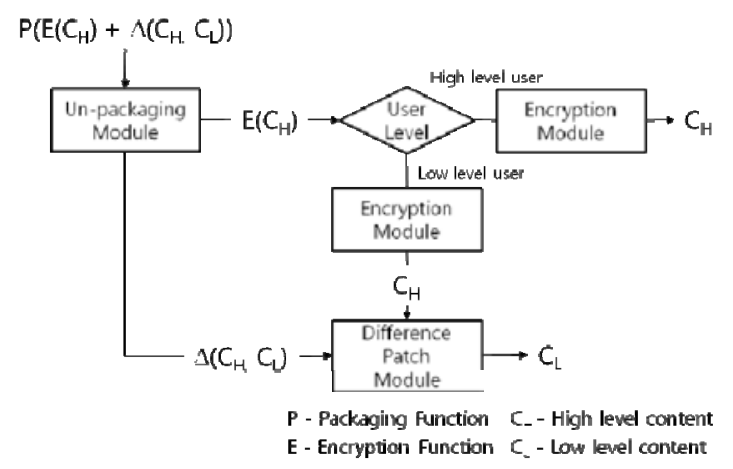

Figure 4. Flow of Multi-Level Access Controller

Figure 4 illustrates the fundamental flow in a Multi-Level Access Controller that controls access, based on access levels. When a user attempts to access packaging $\mathrm{P}(\mathrm{E}(\mathrm{CH})+\Delta(\mathrm{CH}$, $\mathrm{CL})$ ), the Multi-level access controller separately interprets the encrypted $\mathrm{E}(\mathrm{CH})$ and difference extracted $\Delta(\mathrm{CH}, \mathrm{CL})$ through unpackaging module. A high-level user is allowed to access $\mathrm{CH}$ content restored by decrypting $\mathrm{E}(\mathrm{CH})$ depending on user level. In the case of low-level users, they can access to CL content through the decryption of encrypted $\mathrm{E}(\mathrm{CH})$ and difference patch with difference extracted from $\Delta(\mathrm{CH}, \mathrm{CL})$.

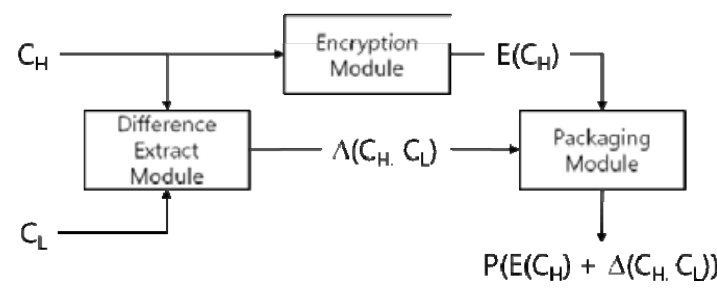

Figure 5. Flow of Multi-Level Access Control packaging

Figure 5 illustrates a fundamental flow in Multi-Level Access Control packaging. The Multi-Level Access Control packager produces Delta $\Delta(\mathrm{CH}, \mathrm{CL})$ by the difference between two input data of High level content $(\mathrm{CH})$ and Low level content (CL). Because $\mathrm{CH}$ is 
confidential information to be protected, it is encrypted using encryption module and then the encrypted $\mathrm{E}(\mathrm{CH})$ and difference extracted from Delta $\Delta(\mathrm{CH}, \mathrm{CL})$ are packaged in the Multi-Level DRM content format. Delta $\Delta(\mathrm{CH}$, CL) data can be protected with encryption according to level of importance.

Figure 6 shows DRM file packaging format to support Multi-level access control. The DCF format [7] of OMA was extended to support all file formats. OMA DRM Container box contains a DCF headers box and protected content box and Delta Compress box was added to support Multi-level access control.

To be suitable to support Multi-Level Delta Compress box, structure of the box needs to be able to hold as many number of multilevels as possible. access to individual layers. Class 0 has access to APA, BDA, EAA, RPG, RPM, TLL and WAA layers. Level 1 has access to APA, BDA, and TLL layers. Level 2 has access to APA, BDA, and EAA layers. Level 3 has access to APA, BDA, RPG and RPM layers. Lastly, Level 4 has access only to APA, BDA layers.

Figure 7 is a visualized image of layers using GIS application in order to check the access control results of layers from class 0 to class 4 based on usage and access levels.

Figure 8 is an image captured for the class 0 and class 3 which has different access control. The protected objects are based on class 0 and class 3s' usage and access levels. In the left figure, objects in the red oval are only granted to a user with access level 0 . If a user with access level 3 attempts to access the protected

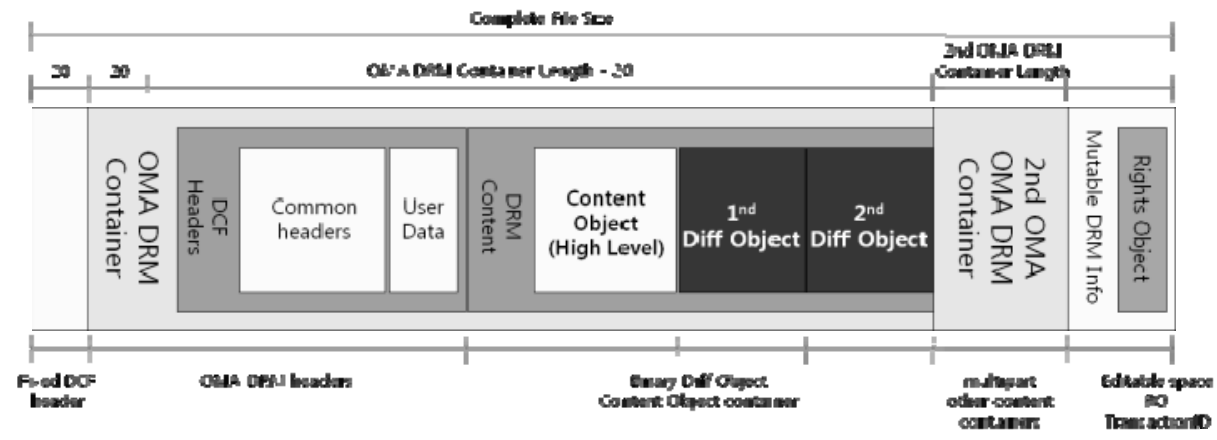

Figure 6. Multi-level access control packaging format

Table 1. Layer information by access level

\begin{tabular}{|c|c|c|c|c|c|c|c|}
\hline \multirow{2}{*}{ Layer Name } & \multirow{2}{*}{ Type } & \multicolumn{7}{|c|}{ Access Level } & \multirow{2}{*}{ Note } \\
\cline { 3 - 6 } & Polygon & 0 & 1 & 2 & 3 & 4 & \\
\hline APA & Polygon & $\mathrm{O}$ & $\mathrm{O}$ & $\mathrm{O}$ & $\mathrm{O}$ & $\mathrm{O}$ & \\
\hline BDA & Polygon & $\mathrm{O}$ & $\mathrm{X}$ & $\mathrm{O}$ & $\mathrm{X}$ & $\mathrm{X}$ & Only class 0 can \\
access to \\
EAA & Polygon & $\mathrm{O}$ & $\mathrm{X}$ & $\mathrm{X}$ & $\mathrm{O}$ & $\mathrm{X}$ & $\mathrm{O}$ \\
\hline RPG & Polygon & $\mathrm{O}$ & $\mathrm{X}$ & $\mathrm{X}$ & $\mathrm{O}$ & $\mathrm{X}$ & protected object \\
\hline RPM & Line & $\mathrm{O}$ & $\mathrm{O}$ & $\mathrm{X}$ & $\mathrm{X}$ & $\mathrm{X}$ \\
\hline TLL & Polygon & $\mathrm{O}$ & $\mathrm{X}$ & $\mathrm{X}$ & $\mathrm{X}$ & $\mathrm{X}$ & \\
\hline WAA & & & & & &
\end{tabular}

\section{Experimental Results}

This section describes the result of Multi-Level Access Controller which controls access of layers, or objects in specific area based on usage and access level of the GIS data. Results of access control are also can be presented as image file of Raster Data.

Table 1 shows the layer information about access levels used in this paper to control objects as shown in the right figure, since a user has no access control to them, it appeared as objects are non-existent. BDA layer is accessible to all levels, but protected object where BDA layer is presented are only accessible to a user with access level 0 . However, the users with other levels except level 0 cannot access the protected objects.

Figure 9 demonstrates the results of the proposed access control to the raster image for access levels of level 0 and lower than level 0 . 


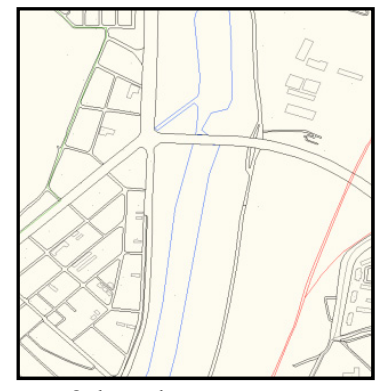

$<0$ level $>$

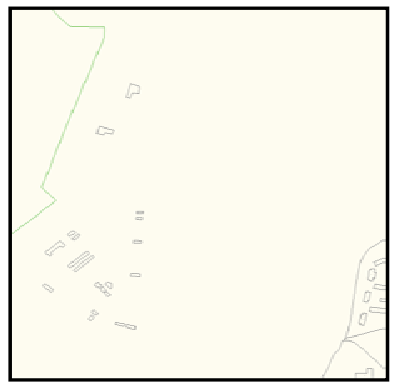

$<2$ level $>$

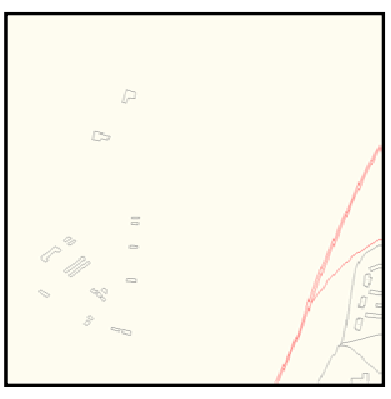

$<1$ level $>$

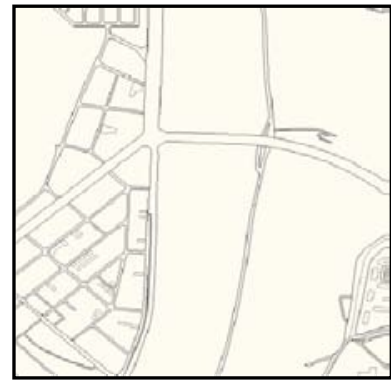

$<3$ level $>$

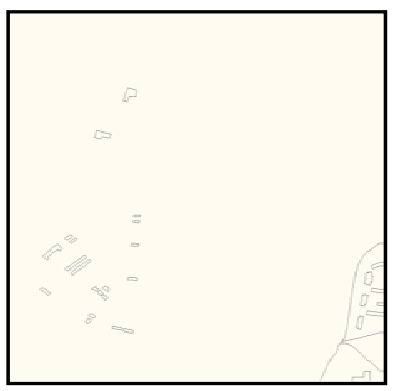

$<4$ level $>$

Figure 7. Access control result of layer units

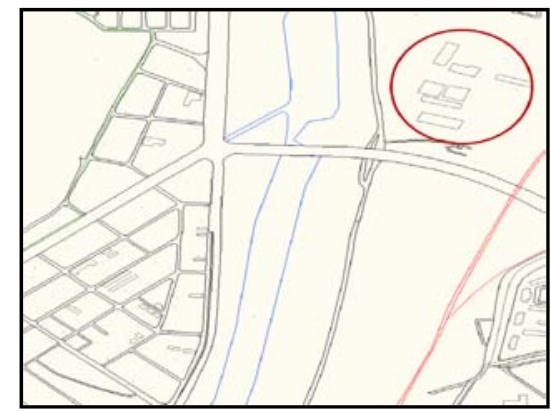

$<0$ level $>$

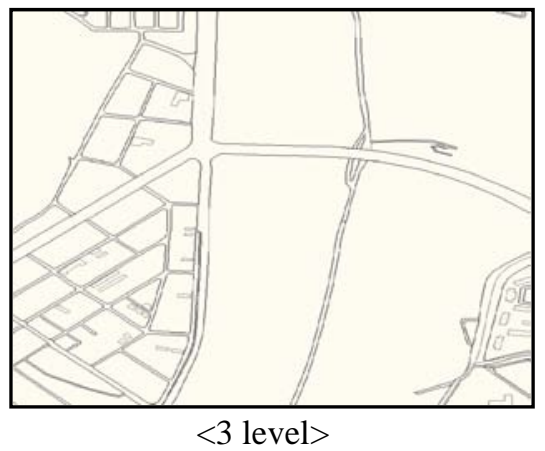

$<3$ level $>$

Figure 8. Access control result of object units

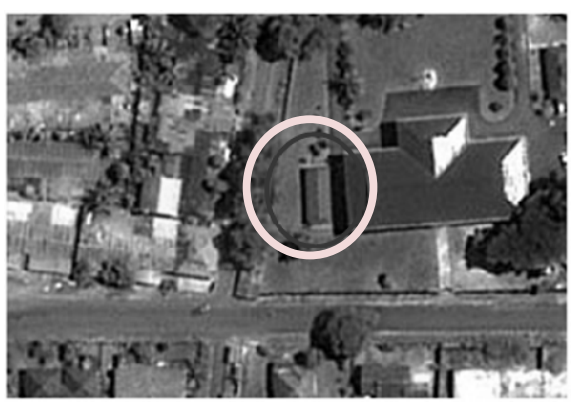

$<0$ level $>$

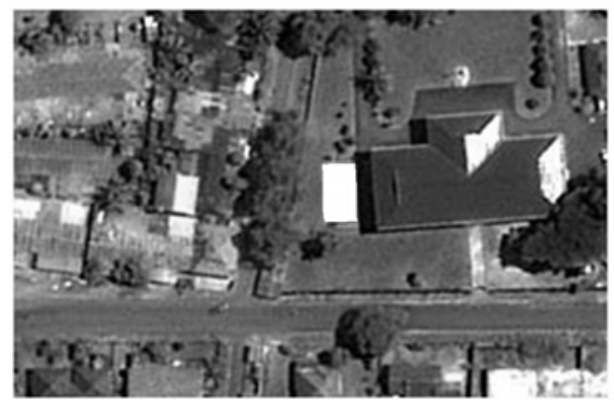

$<$ Lower than 1 level>

Figure 9. Raster image access control result 
The top raster image shows the result when a user with level 0 accessed the object, and the bottom raster image shows the restricted access to building in a red oval when a user with lower than level 0 .

\section{Conclusions}

GIS data is digital content that requires high initial production costs and it is transformed into different digital content according to its usage or access level. For such GIS data a technique is needed for copyright protection and access control based on usage. GIS data is able to control access with Geo-processing technique, but needs an engine which can interpret GIS data file format which supports the access control. This means that an engine should be able to support file formats like Shapefile and Drawing file but the additional work is needed for newly added file format. Image-processing technique is needed for raster image instead of Geo-processing technique. To solve these issues, this paper proposed MultiLevel Access Control technique, Delta Compress Algorithm and DRM content packaging format were proposed as a method to construct Multi-Level Access Controller. Because Multi-Level Access Controller using Delta Compress Algorithm proposed in this paper is applied to all digital content in the same way, the same application is possible for raster image without manipulating the file format. The Multi-Level Access Control is also applicable to the file formats that do not support layer or object concept.

In the future, we will look into methods to improve the performance of Multi-Level Access Control and efficient management systems. Our future work will also include Multi-Level Access Control technique applicable to not only file-based but also streaming-based digital content.

\section{REFERENCES}

1. PARK, J.-H., K.-S. YOON, K.-P. JEON, A Study on Steaming Service for Content Protection, Proceedings of KCAC, 2003, vol. 1, no. 1, pp. 198-201.

2. JEONG, Y., K. YOON, J. RYOU, A Trusted Key Management Scheme for Digital Rights Management, ETRI Journal, Vol.27, No.1, February, 2004.
3. BURNS, R. C., D. D. E. LONG, Efficient Distributed Backup with Delta Compression, Proceedings IOPADS 97, pp. 26-36.

4. MOUNT10 DATA CONTINUITY ARCHITECTS, White Paper FastBIT ${ }^{\text {TM }}$ Binary Patching vs. Block Technology Release: 1.0, October 2004.

5. TRENDAFILOV, D., N. MEMON, T. SUEL, zdelta: An Efficient Delta Compression Tool, Technical Report TRCIS-2002-02, Polytechnic University, CIS Department, June 2002.

6. HEIRBAUT, J. JojoDiff - diff Utility for Binary Files, Available online: http://jojodiff.sourceforge.net/

7. DRM Content Format, Open Mobile Alliance, Approved ver2.1, 2008

8. PARK, S. R., J. S. RUTH LEE, K. W. CHO, K.-C. LEE Multi-level Access Control for Geo-Contents Comm. in Computer and Information Science, 2011, Vol. 256, pp. 183-190

9. SINGH, V. K., R. C. TRIPATHI, Fast and Efficient Region Duplication Detection in Digital Images using SubBlocking Method International Journal of Advanced Science and Technology, 2011, Vol. 35, pp. 93-102.

10. SATHIK, M. M., S. S. SUJATHA, Wavelet based Blind Technique by Espousing Hankel Matrix for Robust Watermarkin International Journal of Advanced Science and Technology, 2011, Vol. 26, pp.57-72.

11. CHA, S.-J., Y.-Y. HWANG, Y.-S. CHANG, K.-O. KIM, K.-C. LEE, Design and Evaluation of Experiment Methods for Improving Performance In GIS Web Services International Journal of Multimedia and Ubiquitous Engineering Vol. 3, No. 1, January 2008.

12. LEE, D. G., A DRM Model for Copyrights Protection based on Hiding Information, International Journal of Multimedia and Ubiquitous Engineering Vol. 1, No. 4, December 2006. 